\title{
Studies on the Electrical and Optical Properties of Magnesium Phthalocyanine Thin Films
}

\author{
T.G. GOPINATHAN and C.S. MENON* \\ School of Pure and Applied Physics \\ Mahatma Gandhi University \\ Kottayam, 686 560, Kerala State
}

Received 25 June 2004; Accepted 10 Sep 2004

\begin{abstract}
Thin films of Magnesium Phthalocyanine (MgPc) are prepared by thermal evaporation technique at a base pressure of $10^{-5} \mathrm{~m}$.bar on thoroughly cleaned glass substrates kept at different constant temperatures. Films of thickness 2400 A.U. coated at room temperature are subjected to post deposition annealing in air by keeping them in a furnace at different constant temperatures, for one hour. The electrical conductivity studies are conducted in the temperature range $300 \mathrm{~K}$ to $525 \mathrm{~K}$. The electrical conductivity is plotted as a function of absolute temperature. The conduction mechanism is observed to be hopping. The thermal activation energy is calculated in different cases and is observed to vary with substrate temperature and annealing temperature. A phase change is observed due to post-deposition annealing at around $523 \mathrm{~K}$. The optical absorption studies are done in the UV-Visible region. The optical band gap energies of the samples are calculated.
\end{abstract}

Key words Magnesium Phthalocyanine, Thermal evaporation, Thin film

\section{Introduction}

Phthalocyanines form an important class of organic semiconductors with a wide range of applications. Metal phthalocyanines are reported as p-type organic semiconductors. Their stability against thermal and chemical decomposition and their intense optical absorption in the visible region of the electromagnetic spectrum have made them very significant candidates for various uses. Since the establishment of semiconduction in phthalocyanines originating with the work of Eby on metal-free phthalocyanine and extension to Copper Phthalocyanine (CuPc) and Magnesium Phthalocyanine (MgPc) by Vartanyan, there has been considerable interest in these materials, related to a variety of possible device fabrications. ${ }^{1}$ They are used as gas sensors, ${ }^{2-5}$ photovoltaic and solar cells, ${ }^{6-10}$ dyes in paint and textile industry, ${ }^{11}$ and opto-electronic devices. ${ }^{12}$ Recently superlattices consisting of organic multilayers have been demonstrated in some combinations of organic materials. ${ }^{13}$ The photoionization energies of $\mathrm{MgPc}$ and CuPc single crystals have been determined in different ambient atmospheres by electrostatic method. ${ }^{14}$ The electrical conductivity of MgPc thin layers are influenced by many gases which made it possible to be used in gas sensing devices. It is observed that the properties of MgPc thin films depend on a number of parameters including the film morphology, which in turn is determined by various preparation parameters. 
It has become apparent that the progress in the development of active materials for molecular electronics based on MgPc thin films require a more comprehensive knowledge of its material properties. In this paper the electrical and optical properties of MgPc thin films coated at different substrate temperatures and of samples subjected to post-deposition annealing at different constant temperatures for one hour are investigated and the thermal activation energies and optical band gap energies are calculated in each case.

\section{Experimental}

Magnesium Phthalocyanine in the powder form (99.99\% pure) obtained from Aldrich $\mathrm{C}$. is used as the source material. The samples are prepared in a "Hind Hi Vac" vacuum coating unit (Model No.12A4). Thermal evaporation technique is used for film preparation. ${ }^{15}$ Thin films are formed on thoroughly cleaned glass substrates kept at room temperature and also kept at different constant temperatures, at a base pressure of $10^{-5} \mathrm{~m}$.bar. The temperature is monitored using a chromel-alumel thermocouple. The film thickness is controlled by a single crystal thickness monitor and are cross-checked by Tolansky's multiple beam interferometry method. ${ }^{16}$ The samples of equal thickness coated at room temperature are annealed in air for one hour in a specially designed furnace, the temperature of which is controlled and recorded by a digital temperature controller cum recorder. The electrical conductivity measurements are done with a Keithley programmable electrometer Model No.617 at a pressure of $10^{-3} \mathrm{~m}$. bar in an approximate temperature range of $300 \mathrm{~K}$ to $525 \mathrm{~K}$. Ohmic contacts are made with silver electrodes and silver paste. The optical transmittance of these thin films in the UV-Visible region is recorded using Shimadzu 160A spectrophotometer.

\section{Results and Discussion.}

The temperature dependence of electrical conductivity is given by the Arrhenius equation [17] $\sigma=\sigma_{0}$ $\exp \left(-E_{T} / 2 k_{B} T\right)$ where $\sigma_{0}$ is the pre-exponential factor, $E_{T}$ is the thermal activation energy, $k_{B}$ is Boltzmann constant and $T$ is the absolute temperature. Such an exponential variation of electrical conductivity is known for semiconductors. Multiple donor levels exist within the forbidden energy gap and the deeper levels can be frozen out as the temperature is increased. Conductivity in phthalocyanines is due to both hopping of holes and charge transport via excited states. In such a case, the conductivity is given by

$\sigma=A \exp \left(-E_{1} / k_{B} T\right)+B \exp \left(-E_{2} / k_{B} T\right)+C \exp \left(-E_{3} / k_{B} T\right)+\ldots$

where $E_{1}$ is the intrinsic energy gap and $E_{2}, E_{3 \ldots}$ are the activation energies corresponding to impurity scattering at comparatively low temperatures. A, B, C are constants. The electrical conductivity of a thin film is given by $\sigma=L / R b t$, where $L$ is the length, $R$ is the resistance, $b$ is the breadth and $t$ is the thickness of the film.

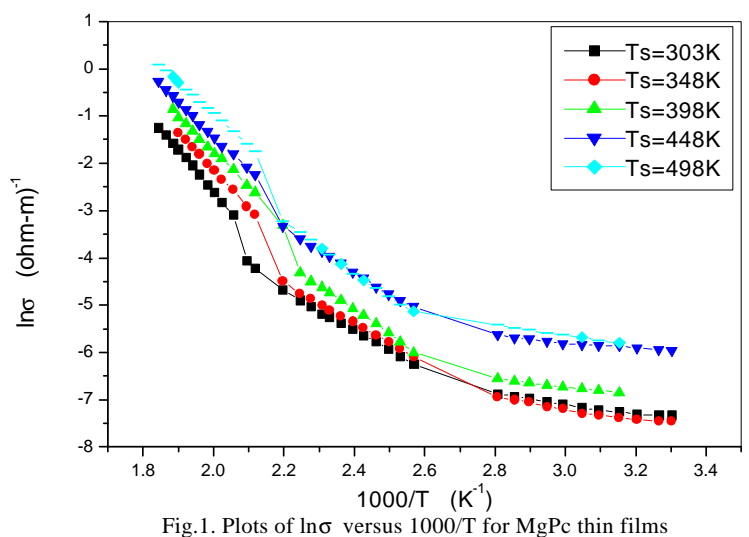

Fig.1. Plots of $\ln \sigma$ versus 1000/T for MgPc thin films coated at different substrate temperatures. 
For MgPc thin films, a plot of In $\sigma$ versus 1000/T shows three linear portions. The higher temperature region is the intrinsic or the non-extrinsic region and the two low temperature regions are the extrinsic regions. From the slopes of the straight line parts of the graphs the thermal activation energies are calculated. Fig.1.gives the plots of In $\sigma$ versus 1000/T for samples coated at different substrate temperatures. The variation of the activation energies when the substrate temperature is increased is given in Table 1.

Table 1. Variation of activation energy for MgPc thin films deposited at various substrate temperatures.

\begin{tabular}{cccc}
\hline \multirow{2}{*}{$\begin{array}{c}\text { Substrate temperature } \\
(\mathrm{K})\end{array}$} & \multicolumn{3}{c}{ Activation Energy $(\mathrm{eV})$} \\
\cline { 2 - 4 } & $\mathrm{E}_{1}$ & $\mathrm{E}_{2}$ & $\mathrm{E}_{3}$ \\
\hline 303 & 0.76 & 0.38 & 0.08 \\
348 & 0.69 & 0.36 & 0.09 \\
398 & 0.66 & 0.43 & 0.08 \\
448 & 0.61 & 0.40 & 0.06 \\
498 & 0.57 & 0.42 & 0.09 \\
\hline
\end{tabular}

It is observed that the thermal activation energy $E_{1}$ corresponding to the intrinsic region decreases with increase in substrate temperature. Higher substrate temperature leads to an increase in the surface energy and to a consequent increase in the grain size of the film. Hence a decrease in the value of activation energy is expected. Also an increase in substrate temperature yields more crystalline thin films with increased electrical conductivity and decreased activation energy. In the two extrinsic regions, the activation energies $E_{2}$ and $E_{3}$ vary in a random manner since in these regions the electrical conductivity is due to impurity scattering.

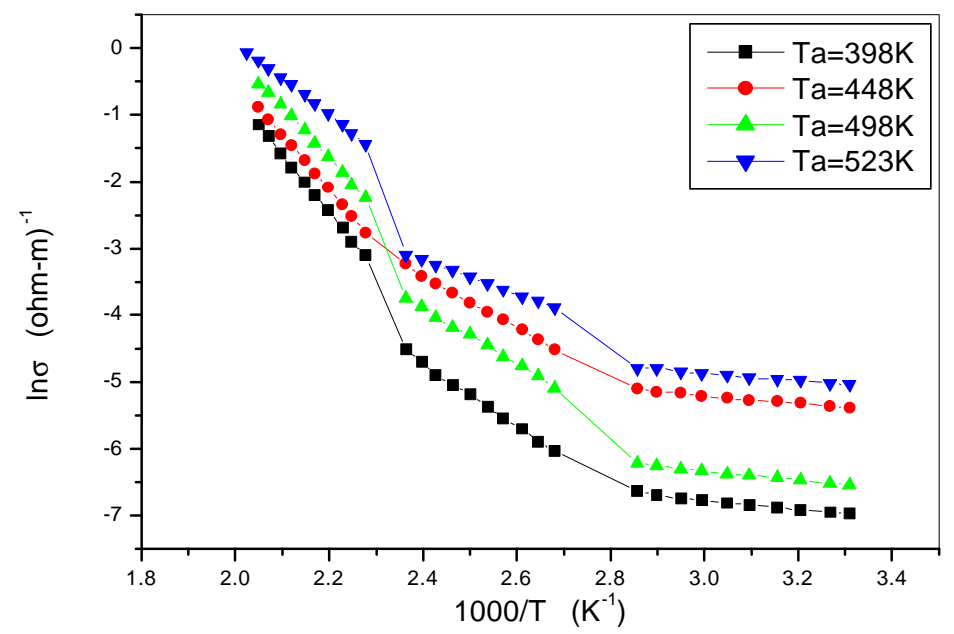

Fig.2. Plots of lno versus 1000/T for MgPc thin films annealed in air at different temperatures.

The steady state transport properties of carriers in organic semiconductors are dominated by the presence and energy distribution of carrier trapping sites. Fig.2.shows plots of In $\sigma$ versus 1000/T for films annealed in air at different constant temperatures, for one hour. The activation energies are calculated in each case. Table 2 shows the variation of activation energy with annealing temperature. 
Table 2. Variation of activation energy for MgPc thin films annealed in air at various temperatures.

\begin{tabular}{cccc}
\hline \multirow{2}{*}{$\begin{array}{c}\text { Annealing } \\
\text { temperature }(\mathrm{K})\end{array}$} & \multicolumn{3}{c}{ Activation Energy $(\mathrm{eV})$} \\
\cline { 2 - 4 } & $\mathrm{E}_{1}$ & $\mathrm{E}_{2}$ & $\mathrm{E}_{3}$ \\
\hline 398 & 0.74 & 0.41 & 0.06 \\
448 & 0.70 & 0.34 & 0.05 \\
498 & 0.65 & 0.36 & 0.06 \\
523 & 0.48 & 0.22 & 0.05 \\
\hline
\end{tabular}

It is found that the activation energy $E_{1}$ corresponding to the intrinsic region decreases with increase in annealing temperature. Due to post-deposition annealing the crystallinity of the film increases and a columnar growth is possible. Also a change in the oxygen impurities present in the sample can take place. These factors can lead to an increase in the conductivity of the film and a decrease in activation energy. In this case also, the activation energies $E_{2}$ and $E_{3}$ corresponding to the extrinsic regions vary in a random manner.

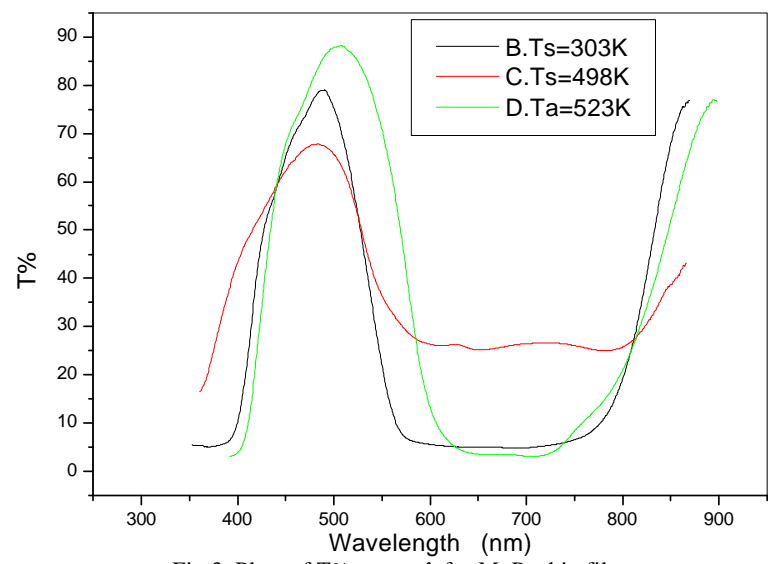

Fig.3. Plots of T\% versus $\lambda$ for MgPc thin films-

B coated at $303 \mathrm{~K}, \mathrm{C}$ coated at $498 \mathrm{~K}$ and D annealed at $523 \mathrm{~K}$

Fig.3. shows plots of percentage of transmission versus wavelength for films prepared at substrate temperatures $303 \mathrm{~K}, 498 \mathrm{~K}$ and for a sample annealed in air at $523 \mathrm{~K}$ for one hour. The film coated at substrate temperature $498 \mathrm{~K}$ gives a larger peak than that at $303 \mathrm{~K}$, showing that film becomes more transparent on increasing the substrate temperature. The marked change in the graph for the sample annealed at $523 \mathrm{~K}$ suggests a possible phase change.

The absorption coefficient $\alpha$ is related to the optical band gap energy $E_{g}$ by the equation $\alpha=\alpha_{0}\left(h v-E_{g}\right)^{1 / 2}$ where $\alpha=(2.303 / t) \log (1 / T)$. Here $t$ is the thickness of the film and $T$ is the transmittance.

Table 3. Calculated Optical band gap energies for MgPc thin films deposited at different substrate temperatures.

\begin{tabular}{|c|c|}
\hline $\begin{array}{c}\text { Substrate } \\
\text { Temperature }(\mathrm{K})\end{array}$ & $\begin{array}{c}\text { Optical Band Gap } \\
\text { Energy (eV) }\end{array}$ \\
\hline 303 & 2.59 \\
\hline 348 & 2.65 \\
\hline 448 & 2.73 \\
\hline 498 & 2.55 \\
\hline
\end{tabular}


Fig.4. gives the plots of $\alpha^{2}$ versus hv for films prepared at different substrate temperatures and Fig.5. shows such curves for air-annealed samples. The optical band gap energies are calculated in each case and are given in Table 3 and 4 respectively.

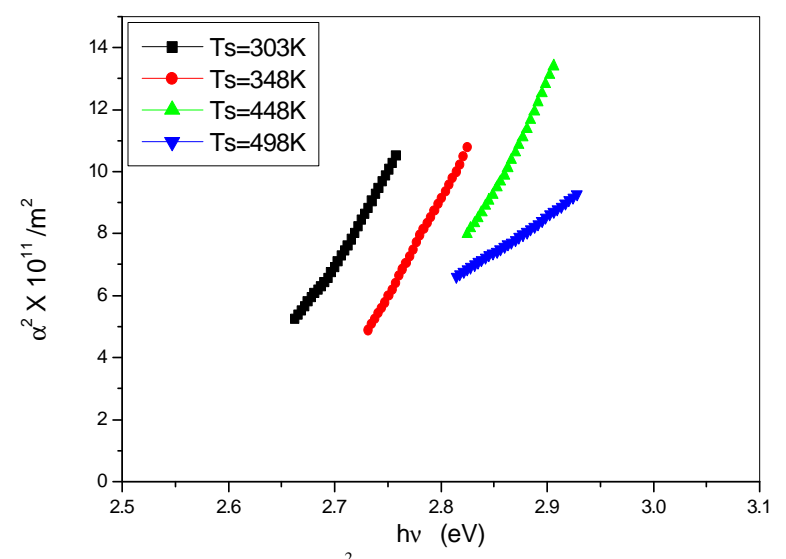

Fig.4. Plots of $\alpha^{2}$ versus hv for $\mathrm{MgPc}$ thin films coated at different substrate temperatures.

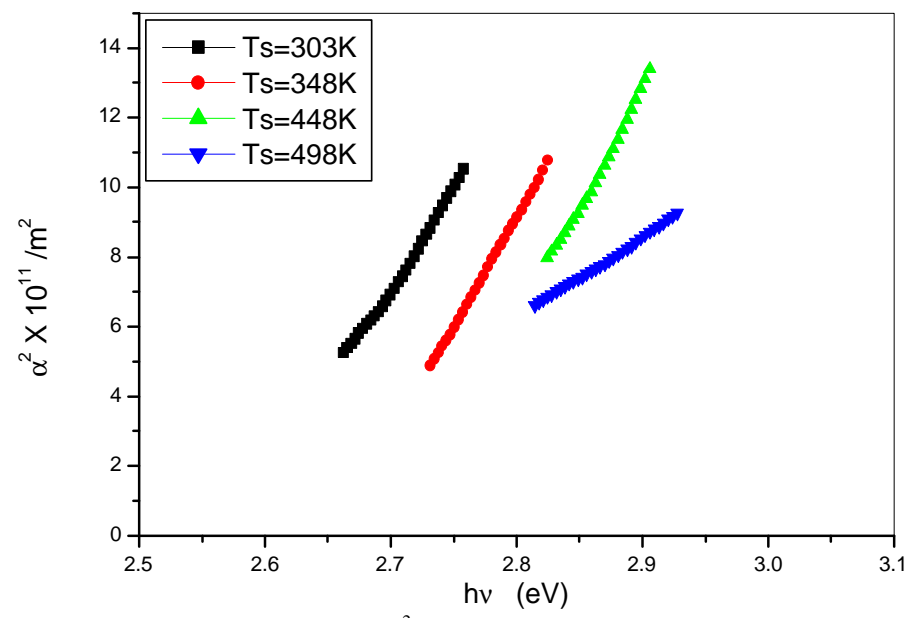

Fig.4. Plots of $\alpha^{2}$ versus $h v$ for $\mathrm{MgPc}$ thin films coated at different substrate temperatures.

Table 4. Calculated Optical band gap energies for MgPc thin films annealed in air at different temperatures.

\begin{tabular}{|c|c|}
\hline $\begin{array}{c}\text { Annealing } \\
\text { Temperature }(\mathrm{K})\end{array}$ & $\begin{array}{c}\text { Optical Band Gap } \\
\text { Energy (eV) }\end{array}$ \\
\hline 398 & 2.60 \\
\hline 448 & 2.58 \\
\hline 498 & 2.59 \\
\hline 523 & 2.56 \\
\hline
\end{tabular}




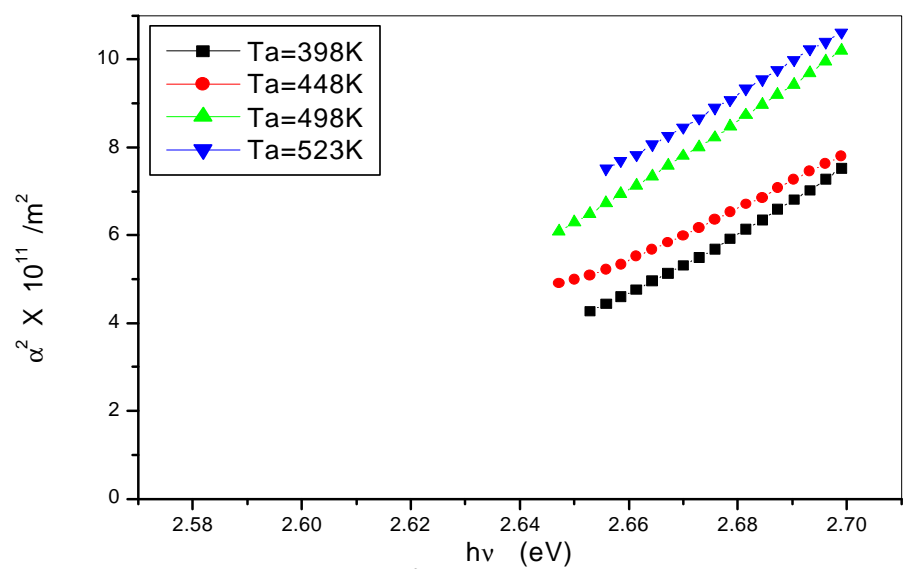

Fig.5. Plots of $\alpha^{2}$ versus hv for $\mathrm{MgPc}$ thin films annealed in air at different temperatures.

\section{Conclusion}

The three-zone Arrhenius plot suggests a transition from intrinsic to extrinsic behaviour of MgPc thin films. The conjugation structure of phthalocyanine is the essential condition leading to non-extrinsic conductivity. The non-extrinsic activation energies of MgPc thin films of thickness 2400 A.U. are found to decrease when the substrate temperature is increased. The two extrinsic activation energies show random changes. For the air annealed samples the intrinsic thermal activation energy is found to decrease with increase in the annealing temperature. The drastic change in the activation energy of the sample annealed in air at $523 \mathrm{~K}$ for one hour suggests a phase transition. The optical band gap energy undergoes no significant variation with difference in annealing temperature.

\section{References}

1. Morel D L and Berger H, J. Appl. Phys. 1975, 46(2), 863.

2. Bott B and Jones J.A, Sens Actuators 1986, 9, 19.

3. Wilson A and Collins R A, Sens Actuators, 1987, 12, 389.

4. Abass A K, Krier A and Collins R A, J. Phys. D. Appl. Phys, 1993, 26, 1120.

5. Abass A. K, Krier A and Collins R A J. Phys. Chem. Solids, 1993, 54, 375.

6. Fanand F.R and Faukner L R, J. Chem. Phys. 1978, 69.

7. Whitlock W J and Panayotatos P, Thin Solid Films 1991, 205, 69.

8. Sharma G D, Synth.Met 1995, 74, 227.

9. Simon J J and Andre H J, Molecular semiconductors; Spinger: Berlin, 1985.

10. Taug, CW, Appl. Phys. Lett. 1986, 48,183.

11. Orti ES, Chem. Phys. 1990, 92, 1228.

12. Honeybonme CL and Ewen R J, J. Phys. Chem. Solids 1983, 44, 831.

13. Shizuo Tokito, Jiro Sakata and Yasunori Taga, J. Appl. Phys. 1995, 77(5),1.

14. Aleyamma George, J. Appl. Phys. 1973, 44, (11), 5148.

15. Chopra K L, Thin Film Phenomena; Mc.Graw Hill; New York, 1985.

16. Ludmila Eckertova, Physics of Thin Films II ed; Plenum Publishing Corporation and SNTL, Prague, 1990.

17. Zabkowska-Waclawek M, Talik P and Waclawek W, Phys Stat Sol 1990,121,489.

18. Ahmad A and Collins R A Thin Solid Films 1992, 217, 75. 


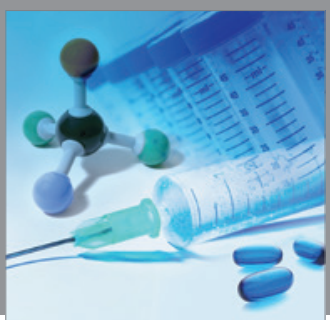

International Journal of

Medicinal Chemistry

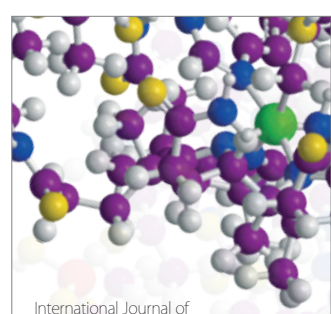

Carbohydrate Chemistry

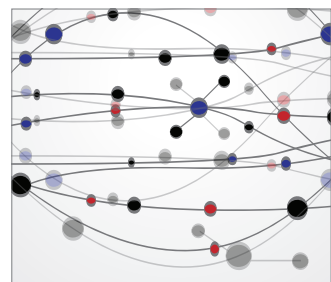

The Scientific World Journal
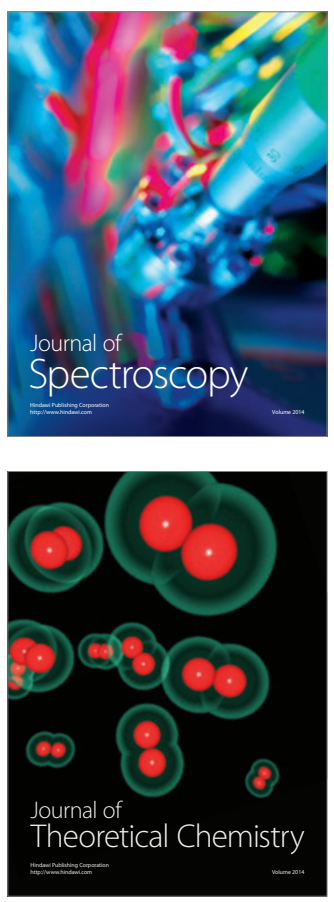
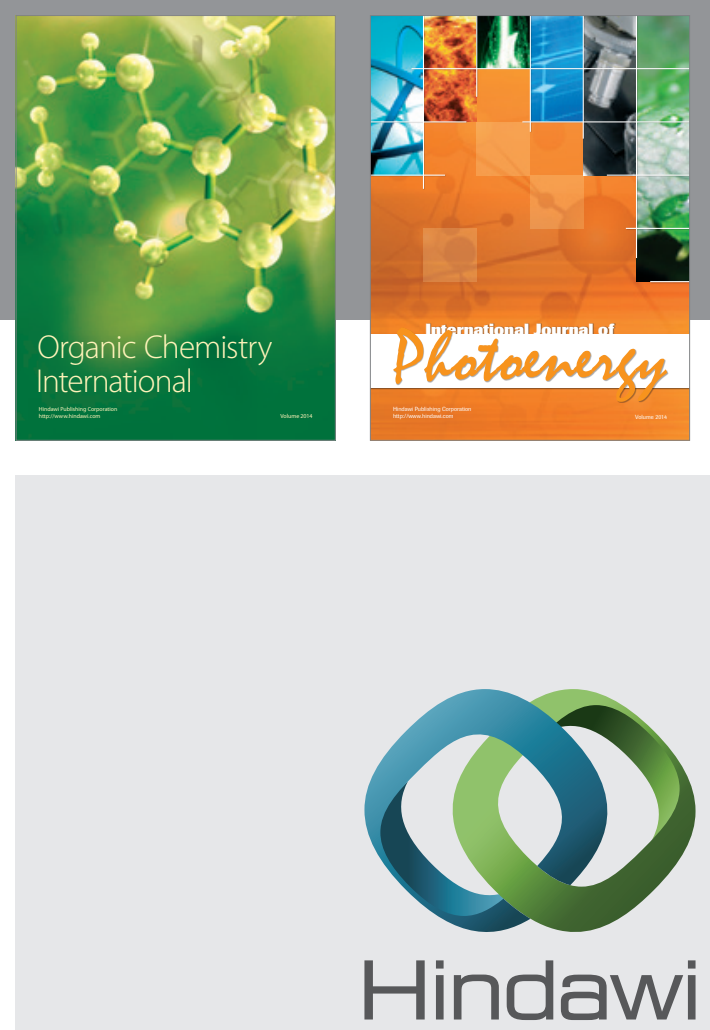

Submit your manuscripts at

http://www.hindawi.com
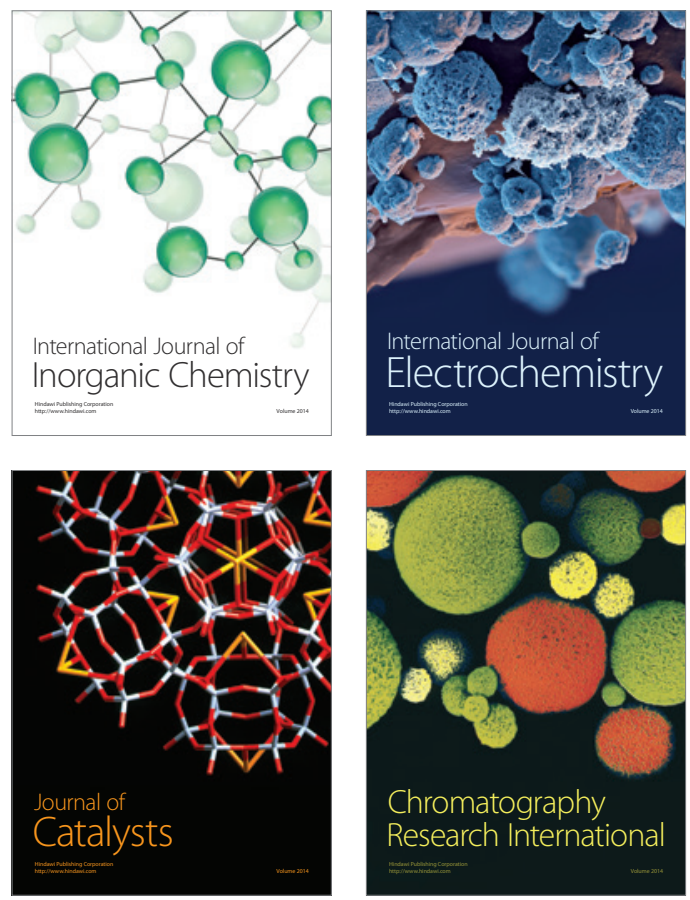
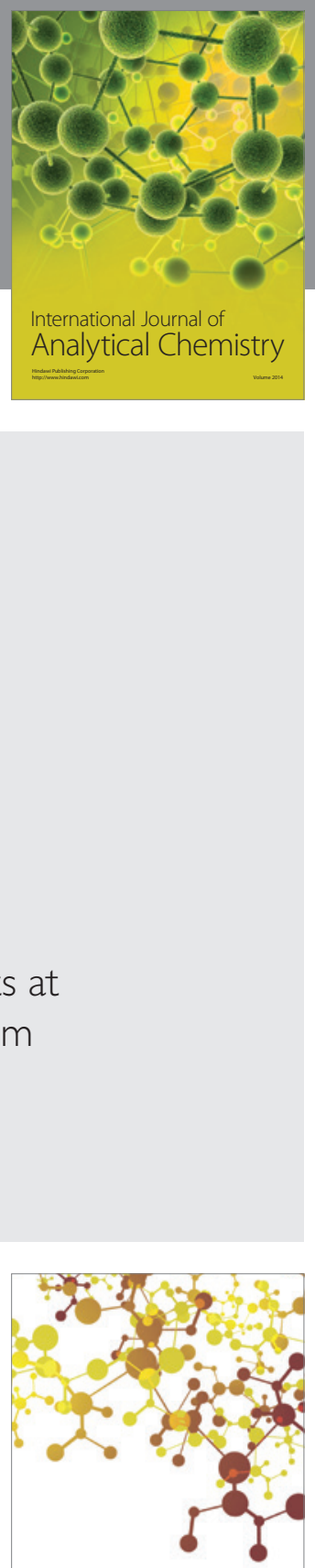

Journal of

Applied Chemistry
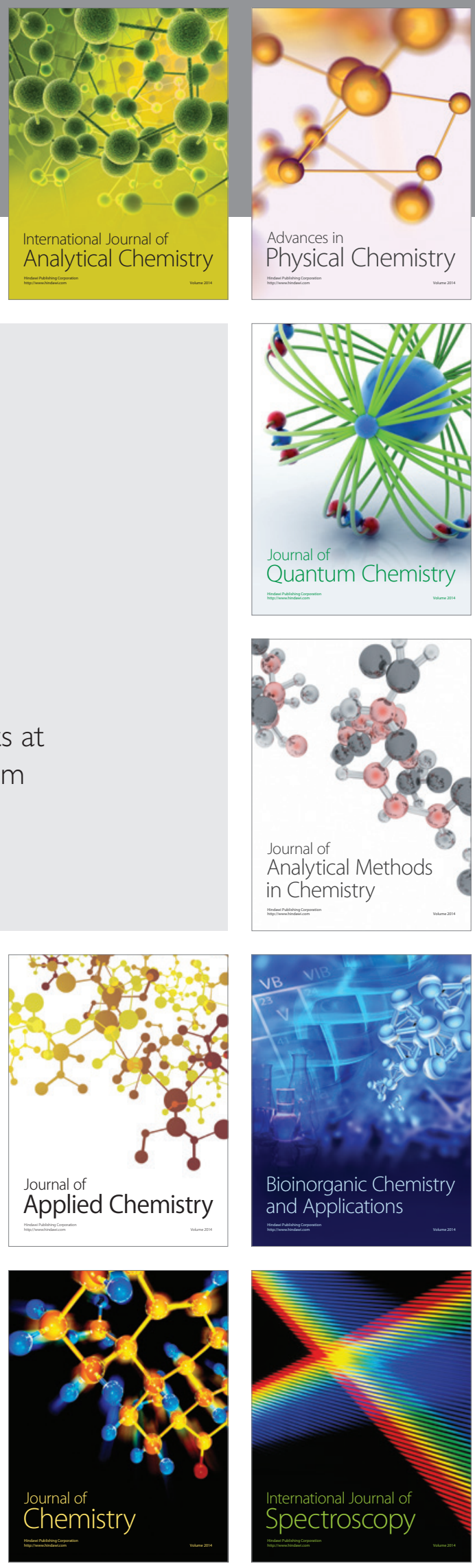